\title{
Updates of the atmospheric dispersion models inside the Local Scale Model Chain of RODOS regarding particles
}

\author{
S. Andronopoulos ${ }^{1}$, T. Schichtel ${ }^{2}$, G. Efthimiou ${ }^{1}$ and J.G. Bartzis ${ }^{3}$ \\ ${ }^{1}$ NCSRD - National Centre for Scientific Research "Demokritos", Institute of Nuclear and Radiological Sciences and Technology, \\ Energy and Safety, 15310 Aghia Paraskevi, Greece. \\ ${ }^{2}$ Institut für Kern- und Energietechnik (IKET), Karlsruhe Institute of Technology (KIT) - Campus North, 76344 Eggenstein- \\ Leopoldshafen, Germany. \\ ${ }^{3}$ Department of Mechanical Engineering, University of Western Macedonia, 50100 Kozani, Greece.
}

\begin{abstract}
New schemes for calculating particles dry and wet deposition have been implemented in the Local Scale Model Chain of RODOS in the frame of Work Package 4 of project PREPARE. Care has been taken not to increase computational times and at the same time, simulations performed with previous deposition schemes to be reproducible. The new schemes take into account particles properties: size and density. Important assumptions adopted so far are that the particles properties are the same for all nuclides and that the properties remain constant during all times of release and dispersion. All formulations adopted for calculating dry and wet deposition coefficients for particles depending on their properties are widely used in the scientific literature. The first tests that have been performed show that the new particles deposition schemes behave as expected and deposition is scaled depending on the size and the density of the particles. The calculated deposition patterns for fine particles with the new scheme are very similar to those calculated by the previous scheme. For larger particles, the differences in comparison to results with the previous deposition scheme justify the implementation of the new scheme in JRODOS.
\end{abstract}

Keywords: particles / particulate matter / atmospheric dispersion / dry deposition / wet deposition / decision support systems

\section{Introduction}

Working Package 4 (WP4) has extended the computational modules in Decision Support Systems by introducing a more accurate modelling of environmental mobility of radioactive particles and therefore more accurate calculation of the related radiation doses. According to the recommendations produced in the project (Andersson, 2015), the Local Scale Model Chain (LSMC) of RODOS has been modified in regards to modelling of dry and wet deposition of particles. Three important requirements for the modelling extensions have been set: (a) not to increase (at least substantially) the computation time of LSMC, (b) to adopt a unified concept for all the Atmospheric Dispersion Models (ADMs) integrated in RODOS/LSMC - this includes the input and output interfaces to other RODOS models as well (e.g., DEPOM/FDMT), and (c) the reproducibility of calculations performed so far by RODOS/ LSMC with the previous dry and wet deposition schemes.

\section{Adopted assumptions}

The particles' size distribution is described inside LSMC by 5 aerosol size "bins" at maximum, following the recommendations of Andersson (2015), regarding the sizes of fuel particles released by severe Nuclear Power Plant accidents and for computational speed requirements. Each particles' size bin is characterised by a characteristic aerodynamic diameter, a mass density and the relative weight in the released activity. The following assumptions are currently adopted for the modelling of particle deposition: (a) the particles physical properties are the same for all radionuclides, (b) the particles' physical properties remain constant in time during release and dispersion, and (c) the deposition-related quantities (dry deposition velocity and washout coefficient) are calculated per size bin and then they are averaged over the size bins to have one value per radionuclide. The latter is a very common approach in dispersion modelling and is made to reduce computational complexity and execution times.

\section{Mathematical formulation of dry and wet deposition}

The detailed mathematical formulation is described by Schichtel et al. (2015). Here a brief outline is given. The dry deposition is calculated through the dry deposition velocity $v_{d}$ that connects the pollutant air concentration near the ground with the dry deposition flux $F_{d}=-v_{d} \times C$. The dry 


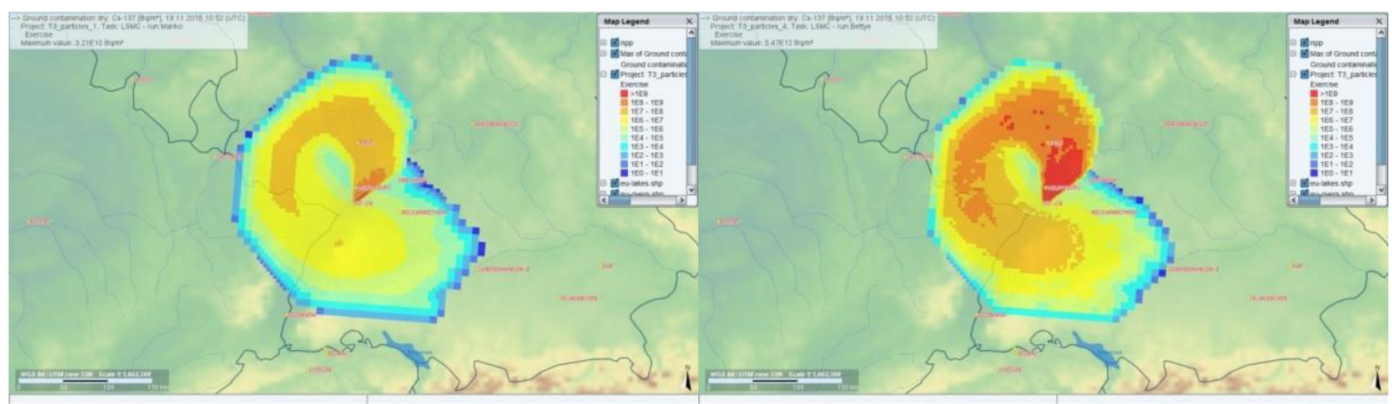

Figure 1. Ground contamination due to dry deposition of particles calculated with the new scheme; left: particles with $A M A D=1 \mu$ m; right: particles with $\mathrm{AMAD}=20 \mu \mathrm{m}$; particles density $2000 \mathrm{~kg} \mathrm{~m}^{-3}$.

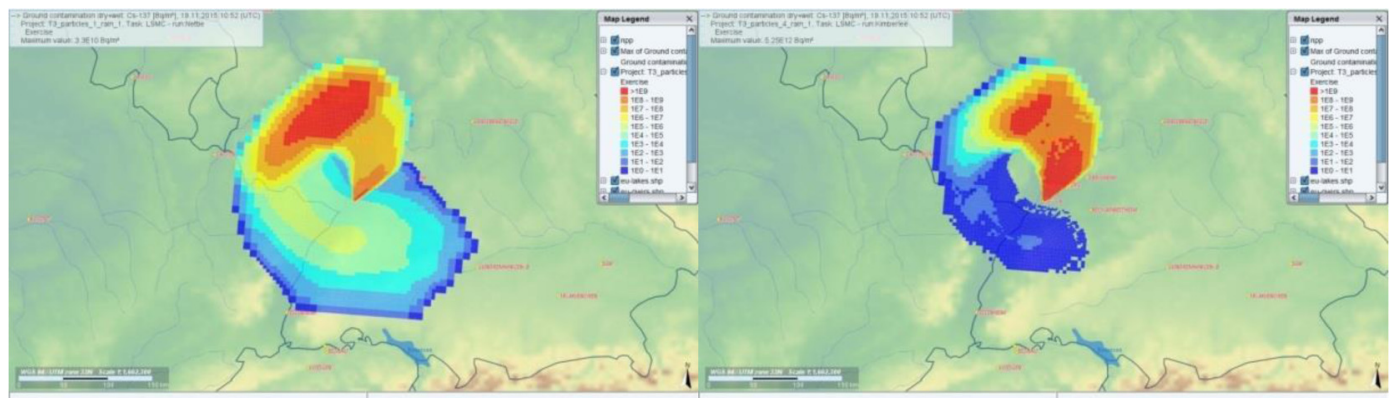

Figure 2. Ground contamination due to wet and dry deposition of particles calculated with the new scheme; left: particles with AMAD = $1 \mu$ m; right: particles with $\mathrm{AMAD}=20 \mu \mathrm{m}$; density $2000 \mathrm{~kg} \mathrm{~m}^{-3}$.

deposition velocity is modelled using the concept of resistances and the following relationship:

$$
v_{d}=\frac{v_{s a} v_{s b} v_{s c}}{v_{s a} v_{s b}+v_{s a} v_{s c}+v_{s b} v_{s c}-v_{s}\left(v_{s a}+v_{s b}+v_{s c}\right)+v_{s}^{2}},
$$

with $v_{s a}:=v_{s}+1 / R_{a}, \quad v_{s b}:=v_{s}+1 / R_{b}, \quad v_{s c}:=v_{s}+1 / R_{c}, R_{a}$ being the "aerodynamic" resistance, $R_{b}$ being the laminar layer resistance, $R_{c}$ being the surface resistance and $v_{s}$ being the sedimentation (or gravitational settling) velocity of particles. The above-mentioned resistances and the sedimentation velocity depend on the atmospheric conditions (wind velocity, atmospheric stability, air temperature), the landcover type and the particles' properties (size-activity median aerodynamic diameter AMAD - and mass density) and are expressed through functions established in the literature, e.g., in Seinfeld (1986). Equation (1) is used to assure a smooth transition from gaseous pollutants to fine aerosols and furthermore to larger particles. It has the correct limiting behaviour: for $v_{s}=0$ reverts to the dry deposition velocity formula for gaseous pollutants. For $R_{c} \rightarrow 0$ reverts to the formula valid for larger particles. In particular, the surface resistance $R_{c}$ for aerosols smaller than $1 \mu \mathrm{m}$ is set to the value used in JRODOS for fine aerosols, for aerosols between 1 and $10 \mu \mathrm{m}$ decreases linearly to zero, and for aerosols larger than $10 \mu \mathrm{m}$ is set to zero. The limit of $10 \mu \mathrm{m}$ size for $R_{c}=0$ is set following Litschke and Kuttler (2008).

Wet deposition is modelled through the wet deposition velocity, which depends on the washout coefficient $\Lambda$. The latter in turn depends on the particles size (the so-called "Greenfield gap"). Following Baklanov and Sørensen (2001) the following approximation of the washout coefficient $\Lambda\left(\mathrm{s}^{-1}\right)$ as a polyno- mial function of particle radius $r(\mu \mathrm{m})$ and rain-rate $q(\mathrm{~mm} / \mathrm{h})$ has been implemented in RODOS/LSMC:

$$
\begin{array}{ll}
\Lambda(r, q)=a_{0} q^{0.79} & r<1.4 \mu \mathrm{m} \\
\Lambda(r, q)=\left(b_{0}+b_{1} r+b_{2} r^{2}+b_{3} r^{3}\right) f(q) & 1.4 \mu \mathrm{m}<r<10 \mu \mathrm{m}, \\
\Lambda(r, q)=f(q) & r>10 \mu \mathrm{m}
\end{array}
$$

with $f(q)=a_{1} q+a_{2} q^{2}$, and $a_{0}, a_{1}, a_{2}, b_{0}, b_{1}, b_{2}, b_{3}$ are empirical constants.

\section{Example calculations}

In this section indicative results are presented from calculations performed with JRODOS LSMC to test the behaviour of the new particles dry and wet deposition schemes. DIPCOT has been used as atmospheric dispersion model. The test case assumed a fictitious 3-hours constant release of Cs-137 which is in particulate form. The total prognosis duration was $24 \mathrm{~h}$. Meteorological conditions were input by hand and consisted of a $2 \mathrm{~m} / \mathrm{s}$ wind, starting with a direction of $225^{\circ}$ (south-west) and rotating counter-clockwise by $45^{\circ}$ every $3 \mathrm{~h}$. Atmospheric stability was assumed variable with stability classes D, B, A, C, D, E, changing every $4 \mathrm{~h}$.

The effect of particles' size on calculated dry deposition with the new scheme is shown in Figure 1. Left part shows the dry deposition patterns for particles with an AMAD of $1 \mu \mathrm{m}$ (fine particles), while right part shows the corresponding patterns for particles of $20 \mu \mathrm{m}$ AMAD. As expected, higher deposition values are calculated for the larger particles, especially in the areas close to the release location. Figure 2 presents ground 
contamination patterns due to total (dry and wet) deposition of particles, to show the effect of particles size on the calculated wet deposition through the new scheme. Wet deposition predictions are inevitably affected by dry deposition because the latter occurs anyway. Therefore total deposition is shown in Figure 2. Rain has been assumed to occur between the 10th and 16th hours of simulation, with intensity ranging from 2 to $3 \mathrm{~mm} / \mathrm{h}$. It is observed that larger particles are strongly deposited closer to the source and the cloud in this case is depleted faster, resulting in less ground contamination in more distant areas.

\section{Conclusions}

New schemes for calculating particles dry and wet deposition have been implemented in RODOS/LSMC. Care has been taken not to increase computational times and at the same time, simulations performed with previous deposition schemes to be reproducible. The new schemes take into account particles' physical properties: size and density. Important assumptions adopted so far are that the particles properties are the same for all nuclides and that the properties remain constant during all times of release and dispersion. The concept adopted for calculating dry and wet deposition coefficients for particles depending on their properties is based on formulations widely used in the scientific literature. The first tests that have been performed show that the new particles deposition schemes behave as expected and deposition is scaled depending on the size and the density of the particles.
The calculated deposition patterns with the new scheme for fine particles are very similar to those calculated by the previous scheme. For larger particles, the differences in comparison to results with the previous deposition scheme justify the implementation of the new scheme in JRODOS.

Acknowledgment. The research leading to these results has received funding from the European Atomic Energy Community Seventh Framework Programme FP7/2012-2013 under grant agreement 323287.

\section{References}

Andersson K.G. (2015) Recommendations for taking into account different physico-chemical forms of contaminants in modelling deposition following a major NPP accident, PREPARE(WP4)(15)-02.

Baklanov A., Sørensen J.H. (2001) Parameterisation of radionuclide deposition in atmospheric long-range transport modelling, Phys. Chem. Earth (B) 26 (10), 787-799.

Litschke T., Kuttler W. (2008) On the reduction of urban particle concentration by vegetation-a review, Meteorolog. Z. 17 (3), 229-240.

Schichtel T., Andronopoulos S., Efthimiou G., Bartzis J.G. (2015) Implementation of PREPARE recommendations for the Atmospheric Dispersion Models inside the Local Scale Model Chain of RODOS, Report PREPARE(WP4)-(15)-03.

Seinfeld J.H. (1986) Atmospheric Chemistry and Physics of Air Pollution. John Wiley \& Sons, New York.

Cite this article as: S. Andronopoulos, T. Schichtel, G. Efthimiou, J.G. Bartzis. Updates of the atmospheric dispersion models inside the Local Scale Model Chain of RODOS regarding particles. Radioprotection 51(HS2), S101-S103 (2016). 